\title{
Questionnaire measures of adult attachment correlate with frontal hemispheric asymmetry in sleep spindle activity
}

Melinda Becske ${ }^{1,2}$, Imre Lázár ${ }^{2,3}$, Róbert Bódizs ${ }^{2,4}$

${ }^{1}$ Department of Psychiatry and Psychotherapy, Semmelweis University, Budapest, Hungary

${ }^{2}$ Institute of Behavioural Sciences, Semmelweis University, Budapest, Hungary

${ }^{3}$ Institute of Social and Communication Sciences, Károli Gáspár University of the Reformed Church in Hungary

${ }^{4}$ Epilepsy Center, National Institute of Clinical Neurosciences, Budapest, Hungary

\section{Correspondence:}

Róbert Bódizs, PhD

Institute of Behavioural Sciences

Semmelweis University

Nagyvárad tér 4.

H-1089 Budapest

HUNGARY 


\section{Abstract}

Introduction: Attachment anxiety and neuroticism were proposed to be associated with relative right frontal neural activity. Since sleep spindles are argued to reflect enhanced offline neuroplasticity, higher spindle activity measured over the right frontal areas relative to the corresponding left frontal ones could index higher attachment anxiety and neuroticism.

Methods: 34 healthy subjects (male $=19 ; \mathrm{M}_{\text {age }}=31.64 ; \mathrm{SD}_{\text {age }}=9.5$ ) were enrolled in our preliminary study. Second night EEG/polysomnography records and questionnaire measures of personality (Zuckermann-Kuhlman Personality Questionnaire) and adult attachment (Relationship Scales Questionnaire) were collected. Frontal slow sleep spindles were measured by the Individual Adjustment Method (IAM), whereas hemispheric asymmetry indexes of spindle occurrence rate, duration and amplitude were derived as normalized left-right differences (electrode pairs: Fp1-Fp2, F3-F4 and F7-F8).

Results: Relative right lateralization of frontolateral and frontopolar slow sleep spindle density and mid-frontal slow spindle duration were associated with attachment anxiety, but spindle lateralization was less closely related to neuroticism. The relationships between frontal slow spindle laterality and attachment anxiety remained statistically significant even after controlling for the effect of neuroticism. The attachment "relationship" dimension (need for close relationships) was related to relative left dominance of frontal slow spindle activation, whereas attachment independence was not correlated with frontal slow spindle lateralization.

Conclusion: Right frontal lateralization of slow sleep spindle activity can potentially serve as a marker for attachment anxiety.

\section{Introduction}

Attachment, as a psychological term introduced by Bowlby, refers to an inborn, biologically based behavioural system that ensures survival of the offspring (Bowlby, 1969). Approach and withdrawal mechanisms drive the behaviour of infants: they tend to move away from objects causing distress and approach their caregivers for comfort (Bowlby, 1969). Individual differences exist in the attachment behaviour, that is referred to as secure and insecure (avoidant or anxious-ambivalent) attachment style (Cassidy \& Shaver, 2016). Avoidant children tend to be more engaged with the environment and less likely to seek proximity of their parents, while ambivalent children seem clingy on their parents and less engaged with the environment (Cassidy \& Shaver, 2016).

Attachment style and emotional regulation are closely related even in adulthood (Gander \& Buchheim, 2015). Adults with secure attachment - having a basic feeling of trust and competence develop better coping strategies, they are emotionally more flexible. It has been argued that they are more willing to explore their own emotions, and can better regulate them (Gander \& Buchheim, 2015). Attachment insecurity, on the other hand, is considered to be a risk factor for developing mental and physical health problems (Cassidy et al., 2013; Cassidy \& Shaver, 2016; Mikulincer \& Shaver, 2012). Insecure attachment style hinders social competence and the ability to form and maintain close relationships (Lee \& Hankin, 2009). Attachment insecurity can have devastating consequences on the individuals' lives by causing them to develop early maladaptive schemas (Bosmans et al., 2020).

Previous research suggest that frontal asymmetry is related to emotional-motivational processes both on trait and state level (J. A. Coan \& Allen, 2004; J. Coan \& Allen, 2003; Hewig, 2018). According to the revised reinforcement sensitivity theory, three basic systems regulate human behaviour that are mostly stable from early childhood throughout the lifetime (Gray \& McNaughton, 2000): 
Behavioural Activation System (related to anticipated positive affect of goal attainment, reward responsiveness and extroversion [Carver \& Scheier, 2008], found to be mediated by left prefrontal areas [Harmon-Jones \& Gable, 2018]), Fight-Flight-Freeze System (fear, negative affect: related to subcortical structures [Dilger et al., 2003]), and a Supervisory control system (revised Behavioural Inhibition System [r-BIS]: related to punishment, nonreward, behavioural inhibition, and risk analysis, control of different motivational urges [Gray \& McNaughton, 2000], mediated by right prefrontal areas [Gable et al., 2018]). The idea of this kind of functional differentiation between the two hemispheres, regarding emotional-motivational processing, is strongly supported by empirical data (including lesion studies [see for review, Gable et al. (2018)]). Lateralization of prefrontal activity is found to be at least partly driven by the dopaminergic system (Nash et al., 2012), it is closely tied to reward processing (Kumar et al., 2014) and reward anticipation (Gorka et al., 2015).

R-BIS is related to effortful control, self-control, the urge to act in a socially desirable manner (Gable et al., 2018). This system controls behaviour by increasing sensitivity towards negative stimuli (Gray \& McNaughton, 2000). Empirical results suggest, that higher resting state left to right frontal asymmetry positively correlates with mental health; e.g. Mikolajczak et al. (2010) found positive correlation between emotional intelligence and left frontal asymmetry, in a study with 31 healthy participants. Relative left frontal dominance during rest correlated with less difficulty in everyday emotional regulation (impulse control) on a sample of 80 adults (Zhang et al., 2020). Left frontal asymmetry was higher during reappraisal of negative images, while there was no difference during suppression in a study recruiting 102 adult participants (Choi et al., 2016).

Individual differences in these basic systems controlling behaviour are regarded stable from early childhood through the lifetime. Functioning of these systems is partly predetermined by genetic factors (e.g. polymorphisms of dopaminergic and serotonergic receptor genes [Gillath et al., 2008]), but is also shaped during childhood as a result of the attachment relationship (Dawson et al., 1992).

A considerable amount of literature has dealt with the question of emotional processing biases related to attachment anxiety - and also with related constructs, e.g. anxiety, social insecurities, social threat and neuroticism - in general, and in terms of frontal hemispheric asymmetry as well (e.g. Moshirian Farahi et al., 2019; Pérez-Edgar et al., 2013; Rognoni et al., 2008). For example, Gartstein et al. (2020) investigated changes in the laterality patterns of infants ( $N=50$ infant-mother pairs) during a mildly stressful task. According to their results, higher left prefrontal activation correlated with more positive affectivity, more synchronous and reciprocal interactions between infants and mothers. They concluded that left prefrontal asymmetry was associated with better emotional control.

Few researchers have addressed the question of frontal asymmetry during sleep so far, and the studies found mixed results (Schmidt et al., 2003; Sikka et al., 2019). Researchers have only investigated hemispheric laterality as a function of alpha electroencephalogram (EEG) asymmetry, assuming that "alpha" reflects cortical inactivity both in wakefulness and during sleep (Schmidt et al., 2003). However, as frequency characteristics of sleep spindling - in fact known to be a marker of cortical reactivation rather than inhibition (Clawson et al., 2016) - overlap with the alpha range, we hypothesize that the question of frontal laterality during sleep has to be re-investigated from a different perspective.

Sleep spindles are burst-like oscillations at the intersection of alpha and beta range occurring during NREM sleep (Lüthi, 2014). Researchers distinguish slow (frontally dominant) and fast (centroparietally located) spindles (Andrillon et al., 2011). Spindling activity is related to neural plasticity and synaptic potentiation: region-specific reactivation of brain circuits, enhanced neuroplasticity that 
facilitates offline memory consolidation, system-level reorganization within functional networks (Clawson et al., 2016).

Evidence suggest that lateralized sleep EEG spindle activity correlates with improvement of highly lateralized skills (motor memory, [Nishida \& Walker, 2007]). Empirical data also suggest that baseline lateralization patterns of sleep spindles are age- and gender-specific, assumed to reflect the functional and anatomical differences in the brain (Bódizs et al., 2017). However, little is known about the relationship of trait-like differences in lateralization of spindling activity and cognitiveemotional dispositions: lateralized psychological functions.

Since lateralization of sleep spindles was found to correlate with asymmetric involvement of hemispheres during online information processing in case of memory tasks (Nishida \& Walker, 2007), we assume that it can also apply to certain trait-like dispositions in cognitive-emotional functioning that appear to show specific lateralization patterns as well (Harmon-Jones \& Gable, 2018; Herrington et al., 2009; Kungl et al., 2016; Moshirian Farahi et al., 2019). We hypothesize that relative left domination of frontal slow spindle lateralization correlates negatively with psychological traits that are argued to be related to enhanced right or reduced left hemispheric frontal activity, namely attachment anxiety and trait-like emotional reactivity indexed by neuroticism.

\section{Methods}

\section{Participants}

Overall 34 healthy adults participated in the study (male $=19 ; \mathrm{M}_{\mathrm{age}}=31.64 ; \mathrm{SD}_{\text {age }}=9.5$ ), however participants with extreme scores (abs $(z$-score) $>3$ ) were excluded from the analysis.

\section{Polysomnographic recordings}

Sleep data was recorded for two consecutive nights in the laboratory by standard polysomnography, including EEG according to the 10-20 system (Jasper, 1958). EEG was recorded form electrodes: Fp1, Fp2, F3, F4, Fz, F7, F8, C3, C4, Cz, T3, T4, T5, T6, P3, P4, O1, O2, and also bipolar EOG above and below the left and the right canthi, respectively, submental EMG, ECG and breathing (thoracic and one abdominal respiratory inductive plethysmography) were measured. The sampling rate of the amplifier was $249 \mathrm{~Hz}$ (precision: 12 bits; hardware prefiltering: 0.5-70 Hz). EEG data were rereferenced off-line to the mathematically linked mastoids [(A1 $+A 2) / 2]$. Sleep EEG recordings of the second nights spent in the laboratory were analyzed. EEG data were manually scored on a 20-s basis by applying standard criteria (Iber et al., 2007). Artifacted epochs were removed on the basis of visual inspection in 4 seconds steps.

\section{Psychometric data}

Dimensions of attachment (anxiety, independence and relationship [need for close relationships]) were measured by the Hungarian adaptation of the Relationship Scales Questionnaire (RSQ-HUN, Csóka et al., 2007). The questionnaire was originally based on Bartholomew's two-dimensional model of adult attachment (Bartholomew \& Horowitz, 1991), but the Hungarian version includes an additional dimension: attachment "relationship". For measuring neuroticism, we administered the Hungarian adaptation of the Zuckermann-Kuhlmann-Aluja Questionnaire (ZKPQ-HUN, [Kövi et al., 
2018]). The questionnaire has a strong biopsychological basis. Items of the neuroticism subscale measure nervousness, worry, anxiety, insecurity and sensitivity. Both are self-report questionnaires.

\section{EEG data analysis}

Spindle activity of all-night NREM sleep was examined with the IAM approach described in previous studies (Bódizs et al., 2009; Ujma et al., 2015). Frequency criteria of slow and fast spindles were based on the individual-specific peaks of spectra between 9-16 Hz (upper and lower frequencies of individual spectral peaks were determined by zero-crossing points of the averaged second order derivatives of the amplitude spectra). Sleep spindle frequencies (fast and slow) were tested for centro-parietal and frontal dominance, respectively. The amplitude criteria were determined in individual- and derivation-specific manner by multiplying the number of intra-spindle frequency bins with the mean amplitude spectrum values corresponding to lower and upper frequency limits. EEG was then band-pass filtered - based on the FFT method - for individual slow and fast sleep spindle frequencies. Envelopes of the filtered signals were calculated. EEG segments meeting the amplitude criteria lasting for at least $0.5 \mathrm{~s}$ were considered spindles. Based on the IAM approach, individualand derivation-specific densities (spindles $\times \mathrm{min}^{-1}$ ), durations (s), and amplitudes $(\mu \mathrm{V}$ ) of slow and fast spindles were calculated.

\section{Statistics}

Since we followed a hypothesis-driven approach, and we were particularly interested in frontal asymmetry during NREM sleep, we chose to examine slow spindle laterality, as slow sleep spindles are known to show a frontal dominance (Gibbs \& Gibbs, 1951; Jobert et al., 1992). Laterality index of slow spindle activity (spindle density, duration and amplitude) was computed with the following equation (laterality index $>0$ indicate greater relative left lateralization, while laterality index $<0$ indicate greater relative right lateralization of sleep spindles):

spindle laterality index $=($ left-right $) /($ left+right $)$

Because of the small sample size and violation of normal distribution, correlations and partial correlations between variables were computed with Spearman's rank correlation coefficient.

\section{Ethics}

Participants signed informed consent for the participation in the study according to the Declaration of Helsinki. The research protocol used in this study was approved by the ethical boards of the Semmelweis University.

\section{Results}

Features of frontal slow spindle lateralization correlated with two out of the three measured attachment dimensions. Attachment anxiety was related to relative right frontal slow spindle 
dominance: i.e. the higher a subject scored on the attachment anxiety dimension, the more right lateralized his/her frontal slow spindles were. This was reflected by the correlations between attachment anxiety and the lateralization of slow spindle density in the lateral frontal and frontopolar electrode sites $\left(\rho_{\text {s AttAnx_vs_F7-F8(SpiDens }}\right)=-0.506, \mathrm{p}<.01 ; \rho_{\text {s AttAnx_vs_Fp1-Fp2(SpiDur) }}=-0.410, \mathrm{p}<$ $.05)$. Attachment anxiety was related to the lateralization of spindle duration in the mid-frontal area $\left(\rho_{s \text { AttAnx_vs_F3-F4(SpiDur) }}=-0.340, \mathrm{p}<.1\right.$ ) and to the lateralization of spindle amplitude in the frontopolar area $\left(\rho_{s \text { AttAnx_vs__Fp1-Fp2(SpiAmp) }}=-0.333, \mathrm{p}<.1\right)$ on a trend level only. Attachment anxiety did not correlate with the other measures of frontal slow spindle laterality (Figure 1, Table 1).
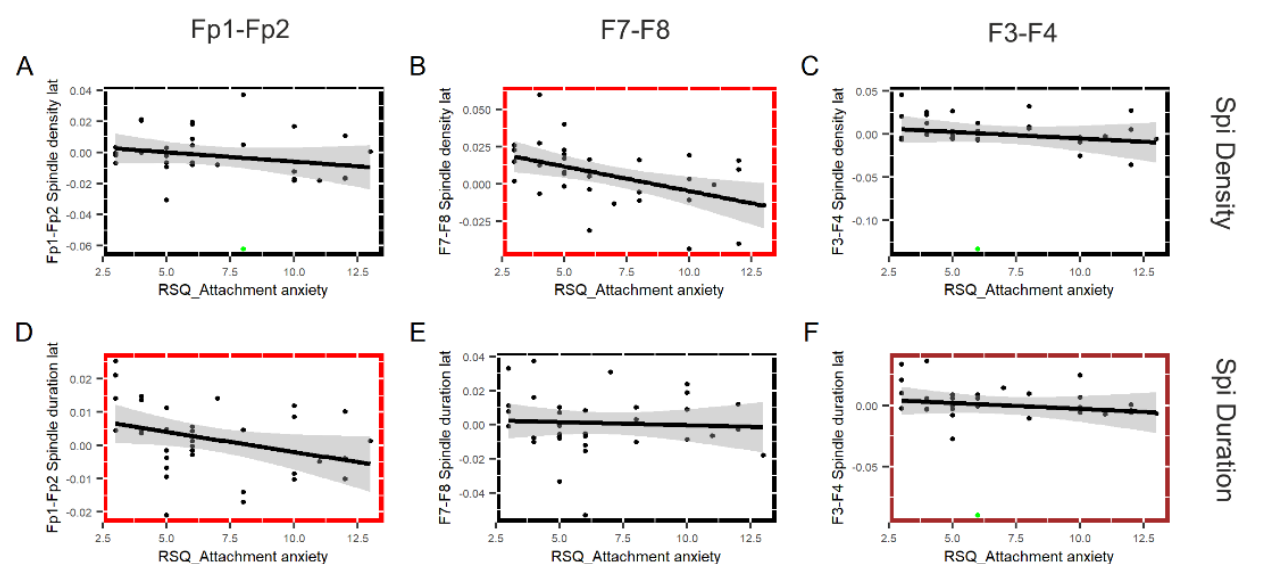

G $\mathrm{H}$
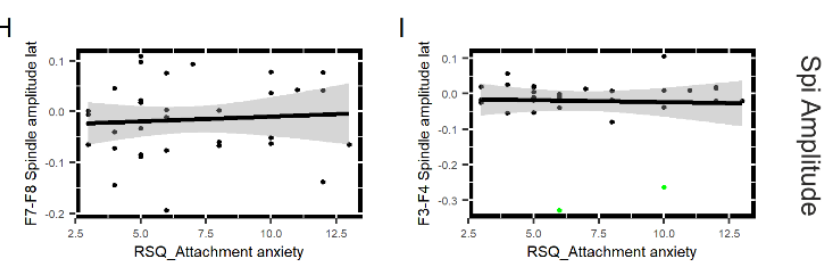

Figure 1. Scatterplots representing the correlations between attachment anxiety and frontal slow sleep spindle lateralization. Black, dark red and light red rectangles indicate non-significant, trend-level ( $p<$ .1) and significant $(p<.05)$ Spearman rank correlations, respectively.

Correlations between attachment independence and frontal slow spindle lateralization were not statistically significant (Figure 2, Table 1). However, the attachment "relationship" dimension was related to relative left spindle dominance in terms of mid-frontal $\left(\rho_{s}\right.$ AttRel_vs_F3-F4(SpiDur) $=0.378, p<.05$ ) and lateral frontal spindle duration $\left(\rho_{\text {s AttRel_vs_F7-F8(SpiDur) }}=0.482, p<.01\right)$ and lateral frontal spindle amplitude $\left(\rho_{\text {s AttRel_vs_F7-F8(SpiAmp) }}=0.378, \mathrm{p}<.05\right.$ [Table 1]). Attachment relationship dimension was not correlated significantly with the other measures of spindle lateralization (Figure 3, Table 1). 

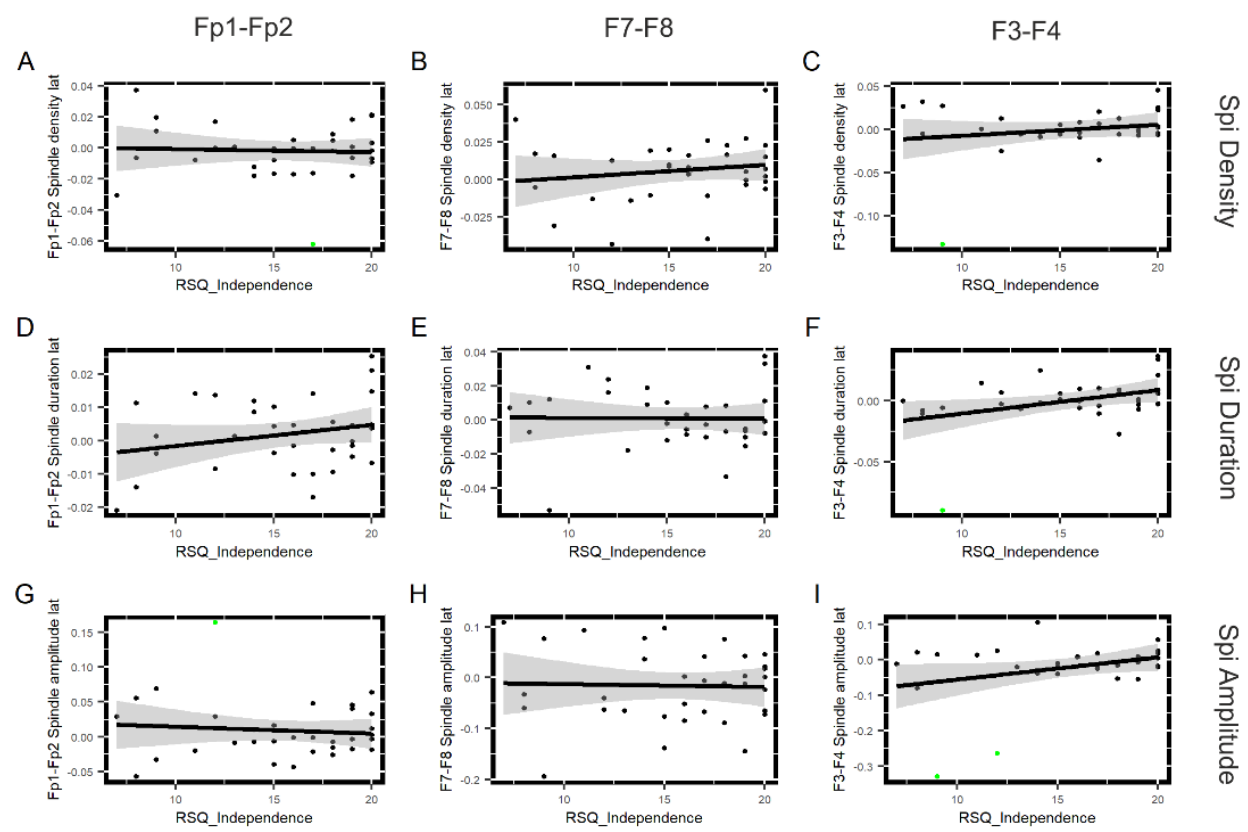

Figure 2. Scatterplots representing the correlations between attachment independence and frontal slow sleep spindle lateralization.
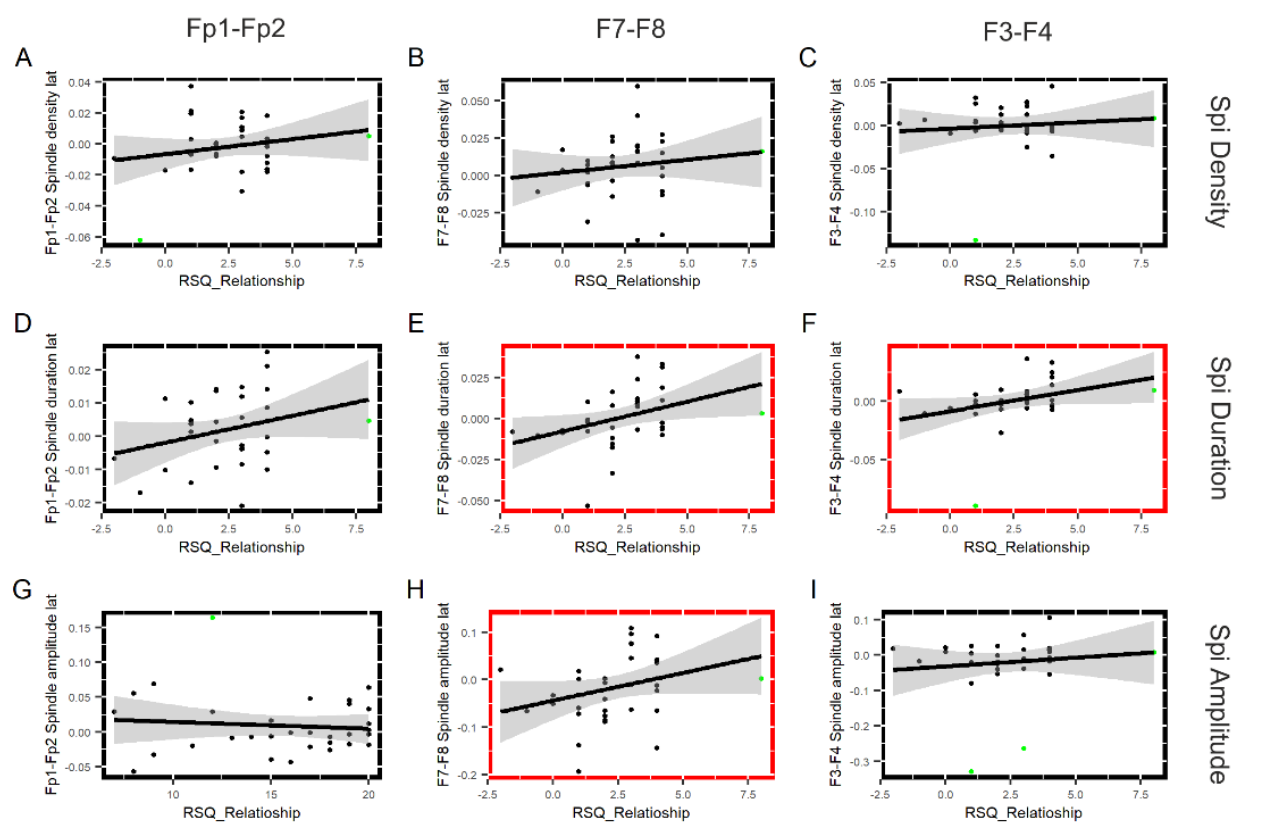

Figure 3. Scatterplots representing the correlations between attachment "relationship" and frontal slow sleep spindle lateralization. Black and light red rectangles indicate non-significant and significant $(p<.05)$ Spearman rank correlations, respectively.

Neuroticism correlated positively with attachment anxiety $\left(\rho_{s \text { Neur_vs_AttAnx }}=0.501 ; p<.01\right)$, and also with the attachment "relationship" dimension $\left(\rho_{s \text { Neur_vs_AttRel }}=0.406 ; \mathrm{p}<.05\right)$. The correlation between neuroticism and attachment independence failed to reach statistical significance, it was only significant on a trend level $\left(\rho_{\text {s Neur_vs_AttInd }}=-0.347 ; p<.1\right.$; Figure 4$)$. 


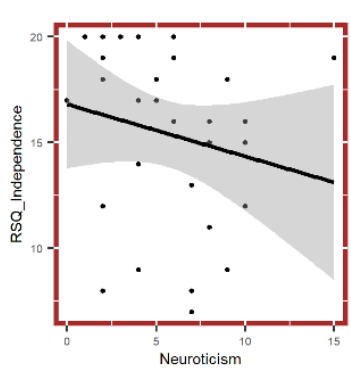

D

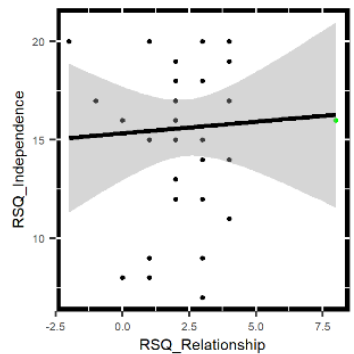

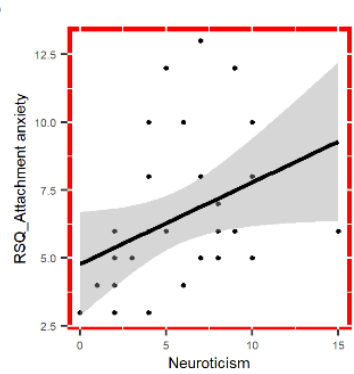

$E$

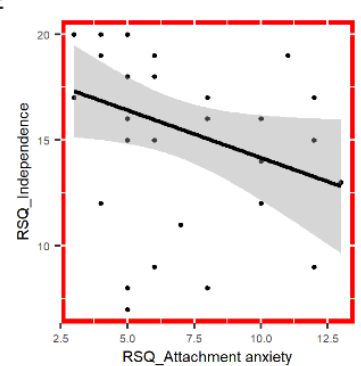

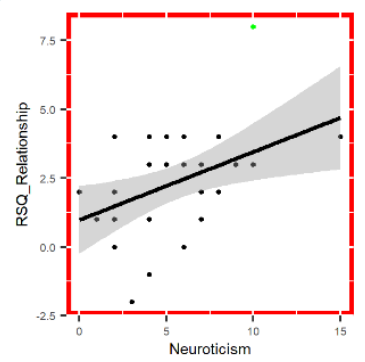

$\mathrm{F}$

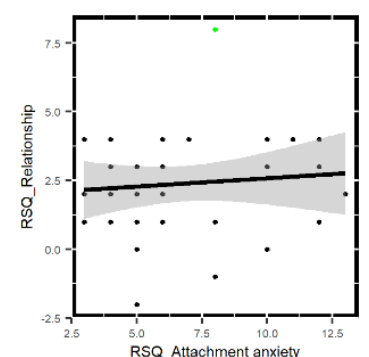

Figure 4. Scatterplots representing the correlations between psychometric variables. Black, dark red and light red rectangles indicate non-significant, trend-level $(p<.1)$ and significant $(p<.05)$ Spearman rank correlations, respectively.

Neuroticism correlated negatively with relative left lateralization of slow sleep spindle amplitude in the frontopolar electrode sites $\left(\rho_{s}\right.$ Neur_vs_Fp1-Fp2/(SpiAmp) $=-0.426, p<.01$ ) but it was not related to the other measures of spindle lateralization at any location (Figure 5, Table 1).

Table 1. Correlations between frontal slow sleep spindle lateralization and psychometric variables (Spearman's $\rho$ )

\begin{tabular}{|c|c|c|c|c|c|c|c|c|c|c|c|c|c|}
\hline $\begin{array}{c}\text { Spearman's Rho } \\
\text { N }\end{array}$ & 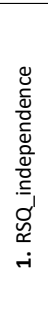 & 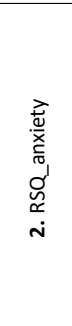 & 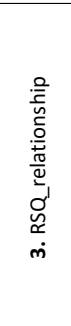 & 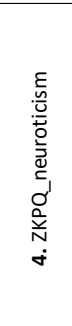 & 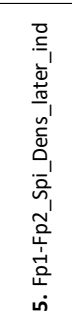 & 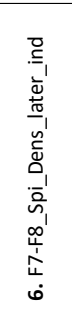 & 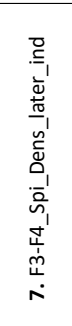 & 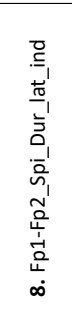 & 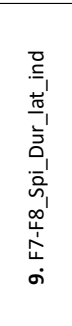 & 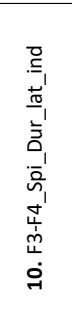 & 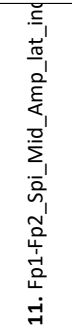 & 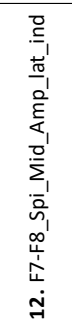 & 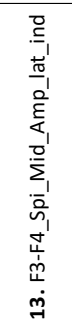 \\
\hline \multirow{2}{*}{ 1. RSQ_indep. } & &,$- 479^{* *}$ & 0.077 & $-.347^{+}$ & 0.095 & 0.124 & 0.009 & 0.124 & -0.106 & 0.277 & 0.125 & -0.038 & 0.089 \\
\hline & & 34 & 33 & 30 & 33 & 34 & 33 & 34 & 34 & 33 & 33 & 34 & 32 \\
\hline \multirow{2}{*}{ 2. RSQ_anx } & & & 0.051 &, $501^{* *}$ & -0.199 &,$- 506 *$ & -0.294 &,$- 410^{*}$ & -0.117 & $-340^{+}$ &,$- 333^{+}$ & 0.108 & -0.022 \\
\hline & & & 33 & 30 & 33 & 34 & 33 & 34 & 34 & 33 & 33 & 34 & 32 \\
\hline \multirow{2}{*}{ 3. RSQ_rel } & & & & $406^{*}$ & -0.029 & 0.181 & $\begin{array}{l}-113 \\
-, 13\end{array}$ & 0.245 & ,482" & $378^{*}$ & -0.051 & $378^{*}$ & 0.114 \\
\hline & & & & 29 & 32 & 33 & 32 & 33 & 33 & 32 & 32 & 33 & 31 \\
\hline \multirow{2}{*}{ 4. ZKPQ_neur } & & & & & 0.182 & -0.098 & -0.238 & $-.309^{+}$ & 0.19 & 0.088 &,$- 426^{*}$ & 0.183 & -0.144 \\
\hline & & & & & 29 & 30 & 29 & 30 & 30 & 29 & 29 & 30 & 28 \\
\hline
\end{tabular}

$\mathrm{p}<0.1$

${ }^{*} p<0.05$

${ }^{* *} p<0.01$ 

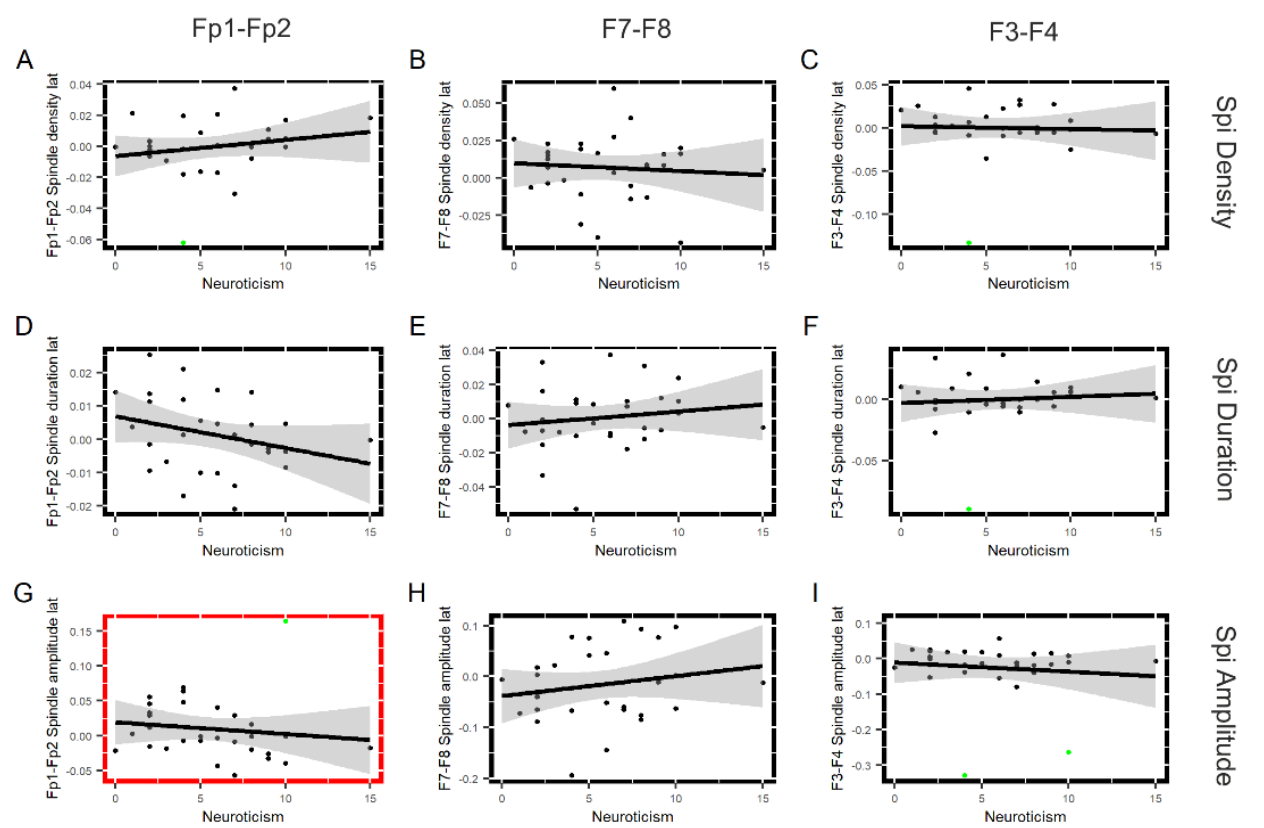

Figure 5. Scatterplots representing the correlations between neuroticism and frontal slow sleep spindle lateralization. Black and light red rectangles indicate non-significant and significant $(p<.05)$ Spearman rank correlations, respectively.

Although, attachment anxiety correlated positively with neuroticism $\left(\rho_{\text {s AttAnx_vs_Neur }}=0.501 ; p<.01\right.$ [Table 1]), most of the above mentioned correlations between attachment and spindle laterality remained statistically significant even after controlling for the effect of neuroticism. Attachment anxiety was clearly related to relative right rather than left frontal slow spindle dominance (lateral frontal spindle density: $\rho_{s}$ AttAn__vs_F7-F8(SpiDens) $=-0.603 ; p<.001$; frontopolar spindle duration: $\rho_{s}$

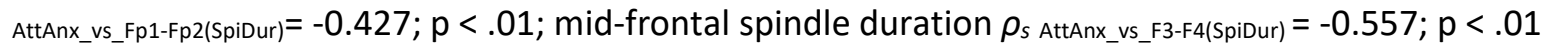
[Table 2]).

Partial correlations also showed that the attachment "relationship" factor correlated with relative left lateralization of frontal slow spindles (lateral frontal spindle density: $\rho_{s}$ AttRel_vs_F7-F8(SpiDens) $=$ 0.494; $\mathrm{p}<.01 ;$ mid-frontal spindle duration: $\rho_{\text {s AttRel_vs_F3-F4(SpiDur) }}=0.442 ; \mathrm{p}<.05$ ) (Table 2). After correcting for the effect of neuroticism, lateralization of frontal slow spindle amplitude did not correlate with the measured dimensions of adult attachment (Table 2).

Table 2. Partial correlations between spindle lateralization and psychometric variables (Spearman's $\rho$, after controlling for the effect of neuroticism) 


\begin{tabular}{|c|c|c|c|c|c|c|c|c|c|}
\hline $\begin{array}{c}\text { Spearman's Rho } \\
\text { (Controlled for } \\
\text { ZKPQ neuroticism) } \\
\mathbf{N}\end{array}$ & 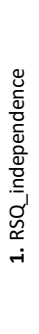 & 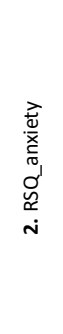 & 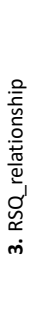 & 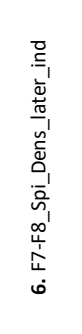 & 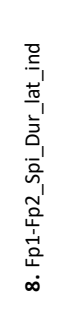 & 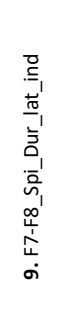 & 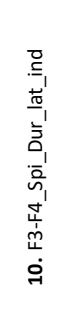 & 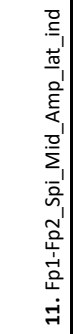 & 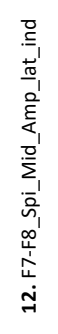 \\
\hline 1. RSQ_indep & & $\begin{array}{c}-.382^{*} \\
30\end{array}$ & & & & & & & \\
\hline 2. RSQ_anx & & & & $\begin{array}{c}-.603^{* * *} \\
30\end{array}$ & $\begin{array}{c}-.427^{*} \\
30 \\
\end{array}$ & & $\begin{array}{c}-.557^{* *} \\
29 \\
\end{array}$ & $\begin{array}{r}-0.172 \\
29 \\
\end{array}$ & \\
\hline 3. RSQ_rel & & & & & & $\begin{array}{c}.494^{* *} \\
29\end{array}$ & $\begin{array}{c}.442^{*} \\
28\end{array}$ & & $\begin{array}{c}0.285 \\
29\end{array}$ \\
\hline
\end{tabular}

\section{Discussion}

In the present study, we aimed to investigate the association between attachment anxiety and the lateralization of frontal slow sleep spindles, examining three features of spindling activity: density, duration and amplitude. Based on the assumptions that 1. regional increase in spindling activity during NREM sleep indicates reactivation of brain areas more actively engaged in neurocognitive functioning at wakefulness (Bódizs et al., 2017; Nishida \& Walker, 2007), and 2. individual differences in baseline spindle lateralization exist (Bódizs et al., 2017), we hypothesized that adult attachment anxiety would be inversely related to relative left domination of frontal slow spindling activity.

The main results of this study indicate that attachment anxiety and relative frontal slow sleep spindle laterality are related indeed, in accordance with our hypothesis, as we found a negative relationship between attachment anxiety score and relative left lateralization of frontal slow sleep spindles in terms of spindle density in the lateral frontal (F7-F8) and frontopolar (Fp1-Fp2) areas. Furthermore, lateralization of spindle duration in the mid-frontal (F3-F4) area and lateralization of spindle amplitude in the frontopolar (Fp1-Fp2) area correlated with the attachment anxiety score on a trend level. This can indicate an overactive right and/or underactive left frontal hemisphere (Cunillera et al., 2014), leading to the over-activation of the Behavioural Inhibition System. That causes an elevated level of trait negative affect (Heym et al., 2008), anxiety (McNaughton et al., 2013), increased risk analysis (Gable et al., 2018) and self-control (Carver \& Connor-Smith, 2010), heightened sensitivity towards negative stimuli, and lower ability to control negative emotional impulses.

As mentioned above, only few studies examined the association between frontal asymmetry during sleep and state or trait affect. Sikka et al. (2019) investigated the relation of alpha asymmetry and state-like dream affect, and found a relationship between dream anger and decreased activity in the right relative to the left frontal hemisphere, based on alpha asymmetry during REM sleep and evening resting wakefulness. Their results can be related to diminished ability to regulate intense emotions or negatively valenced approach-motivational urges during dreaming, as a function of weaker relative right lateralization of frontal cortical activity. Schmidt et al. (2003) on the other hand analyzed the association between frontal alpha asymmetry during sleep and a questionnaire measure of trait motivational style (Behavioral Inhibition Scale). The authors found a moderately strong, negative correlation between the BIS questionnaire score and relative alpha power in the left frontal hemisphere during stage 2 sleep. As greater relative alpha power in the right frontal area 
would indicate relative left dominance in wakefulness, the authors regarded this result as contradictory. However, remembering that frequency characteristics of alpha waves and slow spindles overlap, this result can be straightforwardly interpreted on the ground of current evidence on the function of sleep spindles (Clawson et al., 2016; Nishida \& Walker, 2007).

In addition, we also analyzed the associations of spindle lateralization with the other two dimensions of adult attachment, in order to test whether the lateralization of frontal slow spindles is specifically related to the "anxiety" dimension of the adult attachment questionnaire or it could be more broadly related to different attachment patterns in general. We found that while the "anxiety" dimension was associated with increased relative right dominance of spindling activity, the opposite was true to the "relationship" dimension, as it was related to higher relative left rather than right lateralization of slow spindling activity regarding mid-frontal (F3-F4) and lateral frontal (F7-F8) spindle duration and lateral frontal (F7-F8) spindle amplitude. In simple terms, the more left-dominant a person's frontal slow sleep spindles were, the stronger his/her need for close relationships was according the adult attachment questionnaire. This result is also in accordance with previous data, as relative left frontal dominance is linked to the Behavioral Activation System (Harmon-Jones \& Gable, 2018), associated with higher reward sensitivity, extroversion and trait positive affect (Carver \& Scheier, 2008). The third dimension of the attachment questionnaire, i.e. attachment independence did not seem to correlate with the lateralization of slow spindling activity.

Since, previous research suggests that both attachment issues and laterality of frontal activity can be related to the personality dimension of neuroticism, we tested whether the above relationships remain statistically significant after controlling for the effect of neuroticism. We found that each of the statistically significant correlations remained statistically significant even after partialling out the linear effect of neuroticism, except for one: the "relationship" dimension of adult attachment did not correlate any more with the lateralization of slow spindle amplitude in the lateral frontal area (F7F8). In addition, the trend-level correlation between attachment anxiety and lateralization of slow spindle amplitude in the frontopolar (Fp1-Fp2) area vanished after controlling for neuroticism, but the correlation of attachment anxiety and the lateralization of spindle duration in the mid-frontal (F3-F4) area turned out to be statistically significant only after partialling out the effect of neuroticism. Regarding the relations between neuroticism and adult attachment, neuroticism was found to be positively related to both attachment anxiety and the need for close relationship, but its negative relationship with attachment independence was only significant on a trend level. Contrary to our expectations, neuroticism did not turn out to be closely related to frontal slow spindle lateralization, as it was only significantly correlated to the lateralization of spindle amplitude at the frontopolar (Fp1-Fp2) area.

Lateralization indexes derived from the three features of spindling activity (density, duration and amplitude) correlated differently with questionnaire data: the anxiety dimension correlated with the lateralization of spindle density (in case of one electrode pair) and duration (in one electrode pair), while the "relationship" dimension seemed to be more specifically related to the lateralization of spindle duration, while neuroticism was related to the lateralization of spindle amplitude (in one electrode pair). Based on current knowledge on the subject, it is hard to give an adequate interpretation of this aspect of the results. Furthermore, it also has to be admitted that correlations were not significant at every frontal electrode pair analyzed.

Although this can only be considered a pilot study, the findings suggest that examining the relations between certain psychological factors and lateralization patterns of sleep spindles can be a useful way of gaining more knowledge concerning the relationship of personality and brain activity. As relations of psychological measures and frontal asymmetry are normally estimated based on small 
amounts of EEG data (e.g. few minutes long resting state recordings), analysis of whole night sleep recordings offers an opportunity to gain more robust results (based on more data). Future research might consider investigating the question, whether and how the different features of sleep spindling activity - and the underlying mechanisms behind them - can be specifically related to certain psychological factors, e.g. different dimensions of adult attachment (on larger samples, of course).

\section{Conclusion}

Lateralization of frontal slow sleep spindle activity was found to be weak-to-moderately related to questionnaire measures of adult attachment: attachment anxiety and need for close relationships. The major significance of the study is that it proposes a novel approach to the analysis of sleep EEG data in terms of trait-like emotional dispositions.

\section{Acknowledgements}

Research supported by the Károli Gáspár University of the Reformed Church and the Higher Education Institutional Excellence Program of the Ministry of Human Capacities in Hungary, within the framework of the Neurology thematic program of the Semmelweis University.

\section{References}

Andrillon, T., Nir, Y., Staba, R. J., Ferrarelli, F., Cirelli, C., Tononi, G., \& Fried, I. (2011). Sleep Spindles in Humans: Insights from Intracranial EEG and Unit Recordings. Journal of Neuroscience, 31(49), 17821-17834. https://doi.org/10.1523/JNEUROSCI.2604-11.2011

Bartholomew, K., \& Horowitz, L. M. (1991). Attachment styles among young adults: A test of a fourcategory model. Journal of Personality and Social Psychology, 61(2), 226-244. https://doi.org/10.1037/0022-3514.61.2.226

Bódizs, R., Gombos, F., Ujma, P. P., Szakadát, S., Sándor, P., Simor, P., Pótári, A., Konrad, B. N., Genzel, L., Steiger, A., Dresler, M., \& Kovács, I. (2017). The hemispheric lateralization of sleep spindles in humans. Sleep Spindles \& Cortical Up States, 1(1), 42-54. https://doi.org/10.1556/2053.01.2017.002

Bódizs, R., Körmendi, J., Rigó, P., \& Lázár, A. S. (2009). The individual adjustment method of sleep spindle analysis: Methodological improvements and roots in the fingerprint paradigm. Journal of Neuroscience Methods, 178(1), 205-213. https://doi.org/10.1016/j.jneumeth.2008.11.006 
Bosmans, G., Bakermans-Kranenburg, M. J., Vervliet, B., Verhees, M. W. F. T., \& van IJzendoorn, M. H. (2020). A learning theory of attachment: Unraveling the black box of attachment development. Neuroscience \& Biobehavioral Reviews, 113, 287-298. https://doi.org/10.1016/j.neubiorev.2020.03.014

Bowlby, J. (1969). Attachment and Loss: Attachment. Basic Books.

Carver, C. S., \& Connor-Smith, J. (2010). Personality and coping. Annual Review of Psychology, 61, 679-704. https://doi.org/10.1146/annurev.psych.093008.100352

Carver, C. S., \& Scheier, M. F. (2008). Feedback processes in the simultaneous regulation of action and affect. In Handbook of motivation science (pp. 308-324). The Guilford Press.

Cassidy, J., Jones, J. D., \& Shaver, P. R. (2013). Contributions of Attachment Theory and Research: A Framework for Future Research, Translation, and Policy. Development and Psychopathology, 25(4 0 2), 1415-1434. https://doi.org/10.1017/S0954579413000692

Cassidy, J., \& Shaver, P. R. (Eds.). (2016). Handbook of attachment: Theory, research, and clinical applications (Third edition). Guilford Press.

Choi, D., Sekiya, T., Minote, N., \& Watanuki, S. (2016). Relative left frontal activity in reappraisal and suppression of negative emotion: Evidence from frontal alpha asymmetry (FAA). International Journal of Psychophysiology, 109, 37-44. https://doi.org/10.1016/j.ijpsycho.2016.09.018

Clawson, B. C., Durkin, J., \& Aton, S. J. (2016). Form and Function of Sleep Spindles across the Lifespan. Neural Plasticity, 2016, 1-16. https://doi.org/10.1155/2016/6936381

Coan, J. A., \& Allen, J. J. B. (2004). Frontal EEG asymmetry as a moderator and mediator of emotion. Biological Psychology, 67(1-2), 7-50. https://doi.org/10.1016/j.biopsycho.2004.03.002 Coan, J., \& Allen, J. (2003). The state and trait nature of frontal EEG asymmetry in emotion. The Asymmetrical Brain. 
Csóka Sz., Szabó G., Sáfrány E., Rochlitz R., Bódizs R. (2007). Kísérlet a felnőttkori kötődés mérésére a kapcsolati kérdőív (Relationship Scale Questionnaire) magyar változata. Pszichológia 27(4), 333-355.

Cunillera, T., Fuentemilla, L., Brignani, D., Cucurell, D., \& Miniussi, C. (2014). A Simultaneous Modulation of Reactive and Proactive Inhibition Processes by Anodal tDCS on the Right Inferior Frontal Cortex. PLOS ONE, 9(11), e113537.

https://doi.org/10.1371/journal.pone.0113537

Dawson, G., Klinger, L. G., Panagiotides, H., Spieker, S., \& Frey, K. (1992). Infants of mothers with depressive symptoms: Electroencephalographic and behavioral findings related to attachment status. Development and Psychopathology, 4(1), 67-80.

https://doi.org/10.1017/S0954579400005563

Dilger, S., Straube, T., Mentzel, H.-J., Fitzek, C., Reichenbach, J. R., Hecht, H., Krieschel, S., Gutberlet, I., \& Miltner, W. H. R. (2003). Brain activation to phobia-related pictures in spider phobic humans: An event-related functional magnetic resonance imaging study. Neuroscience Letters, 348(1), 29-32. https://doi.org/10.1016/s0304-3940(03)00647-5

Gable, P. A., Neal, L. B., \& Threadgill, A. H. (2018). Regulatory behavior and frontal activity: Considering the role of revised-BIS in relative right frontal asymmetry. Psychophysiology, 55(1). https://doi.org/10.1111/psyp.12910

Gander, M., \& Buchheim, A. (2015). Attachment classification, psychophysiology and frontal EEG asymmetry across the lifespan: A review. Frontiers in Human Neuroscience, 9. https://doi.org/10.3389/fnhum.2015.00079

Gartstein, M. A., Warwick, H., \& Campagna, A. X. (2020). Electroencephalogram frontal asymmetry changes during emotion-eliciting tasks and parent-child interaction dynamics. Social Development, sode.12484. https://doi.org/10.1111/sode.12484

Gibbs, F., \& Gibbs, E. (1951). Atlas of Electroencephalography, Volume 1. Addison-Wesley Press. 
Gillath, O., Shaver, P. R., Baek, J.-M., \& Chun, D. S. (2008). Genetic correlates of adult attachment style. Personality \& Social Psychology Bulletin, 34(10), 1396-1405. https://doi.org/10.1177/0146167208321484

Gorka, S. M., Phan, K. L., \& Shankman, S. A. (2015). Convergence of EEG and fMRI measures of reward anticipation. Biological Psychology, 112, 12-19. https://doi.org/10.1016/j.biopsycho.2015.09.007

Gray, J. A., \& McNaughton, N. (2000). The neuropsychology of anxiety: An enquiry into the functions of the septo-hippocampal system. Oxford, UK: Oxford University Press.

Harmon-Jones, E., \& Gable, P. A. (2018). On the role of asymmetric frontal cortical activity in approach and withdrawal motivation: An updated review of the evidence. Psychophysiology, 55(1), e12879. https://doi.org/10.1111/psyp.12879

Herrington, J. D., Koven, N. S., Heller, W., Miller, G. A., \& Nitschke, J. B. (2009). Frontal asymmetry in emotion, personality and psychopathology: Methodological issues in electrocortical and hemodynamic neuroimaging. In S. J. Wood, N. B. Allen, \& C. Pantelis (Eds.), The Neuropsychology of Mental Illness (pp. 49-66). Cambridge University Press. https://doi.org/10.1017/СВ09780511642197.008

Hewig, J. (2018). Intentionality in frontal asymmetry research. Psychophysiology, 55(1), e12852. https://doi.org/10.1111/psyp.12852

Heym, N., Ferguson, E., \& Lawrence, C. (2008). An evaluation of the relationship between Gray's revised RST and Eysenck's PEN: Distinguishing BIS and FFFS in Carver and White's BIS/BAS scales. Personality and Individual Differences, 45(8), 709-715. https://doi.org/10.1016/j.paid.2008.07.013

Iber, C., Ancoli-Israel, S., Chesson, A. L., \& Quan, S. (2007). The AASM Manual for the Scoring of Sleep and Associated Events: Rules, Terminology and Technical Specifications. Westchester, IL: American Academy of Sleep Medicine. 
Jobert, M., Poiseau, E., Jähnig, P., Schulz, H., \& Kubicki, S. (1992). Topographical Analysis of Sleep Spindle Activity. Neuropsychobiology, 26(4), 210-217. https://doi.org/10.1159/000118923

Kövi Z., Sándor R., Marcell T., Szabolcs T., Krisztina H., \& András V. (2018). Zuckerman-Kuhlman-Aluja Személyiségkérdőív (ZKA-PQ) magyar verziója. Psychologia Hungarica, 4(1), 23-41.

Kumar, P., Berghorst, L. H., Nickerson, L. D., Dutra, S. J., Goer, F. K., Greve, D. N., \& Pizzagalli, D. A. (2014). Differential effects of acute stress on anticipatory and consummatory phases of reward processing. Neuroscience, 266, 1-12.

https://doi.org/10.1016/j.neuroscience.2014.01.058

Kungl, M. T., Leyh, R., \& Spangler, G. (2016). Attachment Representations and Brain Asymmetry during the Processing of Autobiographical Emotional Memories in Late Adolescence. Frontiers in Human Neuroscience, 10. https://doi.org/10.3389/fnhum.2016.00644

Lee, A., \& Hankin, B. L. (2009). Insecure Attachment, Dysfunctional Attitudes, and Low Self-Esteem Predicting Prospective Symptoms of Depression and Anxiety During Adolescence. Journal of Clinical Child and Adolescent Psychology: The Official Journal for the Society of Clinical Child and Adolescent Psychology, American Psychological Association, Division 53, 38(2), 219-231. https://doi.org/10.1080/15374410802698396

Lüthi, A. (2014). Sleep Spindles: Where They Come From, What They Do. The Neuroscientist, 20(3), 243-256. https://doi.org/10.1177/1073858413500854

McNaughton, N., Swart, C., Neo, P., Bates, V., \& Glue, P. (2013). Anti-anxiety drugs reduce conflictspecific "theta"-A possible human anxiety-specific biomarker. Journal of Affective Disorders, 148(1), 104-111. https://doi.org/10.1016/j.jad.2012.11.057

Mikolajczak, M., Bodarwé, K., Laloyaux, O., Hansenne, M., \& Nelis, D. (2010). Association between frontal EEG asymmetries and emotional intelligence among adults. Personality and Individual Differences, 48(2), 177-181. https://doi.org/10.1016/j.paid.2009.10.001

Mikulincer, M., \& Shaver, P. R. (2012). An attachment perspective on psychopathology. World Psychiatry, 11(1), 11-15. 
Moshirian Farahi, S. M., Asghari Ebrahimabad, M. J., Gorji, A., Bigdeli, I., \& Moshirian Farahi, S. M. M. (2019). Neuroticism and Frontal EEG Asymmetry Correlated With Dynamic Facial Emotional Processing in Adolescents. Frontiers in Psychology, 10, 175. https://doi.org/10.3389/fpsyg.2019.00175

Nash, K., Inzlicht, M., \& McGregor, I. (2012). Approach-related left prefrontal EEG asymmetry predicts muted error-related negativity. Biological Psychology, 91(1), 96-102. https://doi.org/10.1016/j.biopsycho.2012.05.005

Nishida, M., \& Walker, M. P. (2007). Daytime Naps, Motor Memory Consolidation and Regionally Specific Sleep Spindles. PLoS ONE, 2(4), e341. https://doi.org/10.1371/journal.pone.0000341

Pérez-Edgar, K., Kujawa, A., Nelson, S. K., Cole, C., \& Zapp, D. J. (2013). The relation between electroencephalogram asymmetry and attention biases to threat at baseline and under stress. Brain and Cognition, 82(3), 337-343. https://doi.org/10.1016/j.bandc.2013.05.009

Rognoni, E., Galati, D., Costa, T., \& Crini, M. (2008). Relationship between adult attachment patterns, emotional experience and EEG frontal asymmetry. Personality and Individual Differences, 44(4), 909-920. https://doi.org/10.1016/j.paid.2007.10.021

Schmidt, L. A., Cote, K. A., Santesso, D. L., \& Milner, C. E. (2003). Frontal Electroencephalogram Alpha Asymmetry During Sleep: Stability and Its Relation to Affective Style. Emotion, 3(4), 401-407. https://doi.org/10.1037/1528-3542.3.4.401

Sikka, P., Revonsuo, A., Noreika, V., \& Valli, K. (2019). EEG Frontal Alpha Asymmetry and Dream Affect: Alpha Oscillations over the Right Frontal Cortex during REM Sleep and Presleep Wakefulness Predict Anger in REM Sleep Dreams. The Journal of Neuroscience, 39(24), 47754784. https://doi.org/10.1523/JNEUROSCI.2884-18.2019

Ujma, P. P., Gombos, F., Genzel, L., Konrad, B. N., Simor, P., Steiger, A., Dresler, M., \& Bódizs, R. (2015). A comparison of two sleep spindle detection methods based on all night averages: Individually adjusted vs. fixed frequencies. Frontiers in Human Neuroscience, 9, 52. https://doi.org/10.3389/fnhum.2015.00052 
Zhang, J., Hua, Y., Xiu, L., Oei, T. P., \& Hu, P. (2020). Resting state frontal alpha asymmetry predicts emotion regulation difficulties in impulse control. Personality and Individual Differences, 159, 109870. https://doi.org/10.1016/j.paid.2020.109870 